\title{
vbvs.concurrent: Fitting Methods for the Functional Linear Concurrent Model
}

\section{DOI: $10.21105 /$ joss. 00141}

\section{Software}

- Review ¿

- Repository ca

- Archive ¿

\section{Licence}

Authors of JOSS papers retain copyright and release the work under a Creative Commons Attribution 4.0 International License (CC-BY).

\section{Jeff Goldsmith ${ }^{1}$}

1 Columbia University

\section{Summary}

Functional data analysis is concerned with understanding measurements made over time, space, frequencies, and other domains for multiple subjects. Given the ubiquity of wearable devices, it is common to obtain several data streams monitoring blood pressure, physical activity, heart rate, location, and other quantities on study participants in parallel. Each of these data streams can be thought of as functional data, and the functional linear concurrent model is useful for relating predictor data to an outcome. This model can be written

$$
Y_{i}(t)=\beta_{0}(t)+\sum_{k=1}^{p} X_{i k}(t) \beta_{k}(t)+\delta_{i}(t)
$$

where $Y_{i}(t)$ is the functional response for subject $i$, the $X_{i k}(t)$ are functional predictors, the $\beta_{k}(t)$ are functional coefficients of interest, and the $\delta_{i}(t)$ are possibly correlated errors. This package implements two statistical methods (with and without variable selection) for estimating the parameters in the functional linear concurrent model; these methods are described in detail (Goldsmith and Schwartz 2017).

Given tidy datasets containing functional responses and predictors for all subjects, vb_concurrent and vbvs_concurrent fit the functional linear concurrent model using variational Bayes and variational Bayes with variable selection, respectively. These functions produce objects of class $f l \mathrm{~cm}$ and have the same structure. Coefficients and predictions can be extracted or computed from flmc objects using coef and predict. Interactive visualizations of flmc objects are supported through the refund.shiny package (Wrobel and Goldsmith 2015, Wrobel et al. (2016)).

\section{References}

Goldsmith, J, and J E Schwartz. 2017. "Variable Selection in the Functional Linear Concurrent Model." Under Review.

Wrobel, J, and J Goldsmith. 2015. Refund.shiny: Interactive Plotting for Functional Data Analyses.

Wrobel, J, S-Y Park, A-M Staicu, and J Goldsmith. 2016. "Interactive Graphics for Functional Data Analyses." Stat 5: 88-101. doi:10.1002/sta4.109. 